\title{
Periodization of nutrition in cycling: something basic!!!
}

\author{
Raúl López-Grueso ${ }^{1,2} \bowtie$
}

Cycling is characterised by being an individual sport that is carried out as a team and where the intensity and duration is relatively variable, from minutes at maximum to long hours, with different flat/mountain courses and altitudes, and in climatic conditions that are not always pleasant (whether heat or cold, dry or humid). Or all of them together, e.g. Tour de France (Sanders \& Heijboer, 2019).

However, each cyclist's body must be as efficient as possible in order to perform adequately in each of these situations, to their own benefit or for the best-placed partner (Faria et al, 2005).

That is why, to reach the highest level you have to train in different conditions, situations, and of course, taking into account what you will find in competition and what not (Seiler, 2010; Stöggl and Sperlich, 2015).

For this purpose, if you train and compete differently ... you will have to feed yourself in a different way. It is the importance of the nutrient timing (Kerksick et al, 2017; Jeukendrup, 2017; Stellingwerff et al, 2019).

It is the maxim that today coaches, dietitians and physiologists insist and try to communicate: nutrition/hydration must help achieve the metabolic goals, health and performance that the cyclist wants to accomplish and that their level is maximised based on their potential

Focusing the physiological adaptation on the cyclist, uniting training load and pre-during-post consumption, will be utmost, obviously preserving the state of health (Martin et al, 2002). Similar patterns are shown by runners/walkers that can be practiced with cyclists (Heikura et al, 2018), especially in single-day (Heikura et al, 2019) or multi-stage (Muros et al, 2019; SánchezMuñoz et al, 2016) races.

Without health, performance will be limited. And, in terms of energy intake/expenditure, it is especially important when trying to combine an improvement in body composition (usually, by decreasing body fat and preserving muscles), and having sufficient amounts of energy substrates for when pedalling at high intensity, but not for it, causing damage to health.

Carbohydrate $(\mathrm{CHO})$ periodization is key for high intensity efforts, either seeking physiological adaptation or to perform in training (and competition, of course). Hence, "a standard diet, high or low in carbohydrates" is not always accepted as valid for the entire season (Burke and Hawley, 2018).
It will be the work of the dietitian and physical trainer, in harmony with the cyclist and the coaching staff, who will decide which objectives to develop at what period of the year.

The training is programmed with a purpose. The eating helps to achieve it. And, in the attempt to maximise the $\mathrm{CHO}$ consumption when the intensity is high, but also to use the metabolism of fats, several subjects are considered as possibly beneficial.

Gejl et al (2017) observed how to perform highintensity cycling exercise in the morning and 2 hours of moderate intensity in the afternoon, with high $\mathrm{CHO}$ $(415 \mathrm{~g})$ or low CHO (80 g) isocaloric diet, 3 days a week for 4 weeks, can increase performance in both groups (improvement of VO2max, 30-min Time Trial), but does not cause adaptations of muscle glycogen content, enzymatic activities (phosphorylated acetylCoA carboxylase -pACC- and citrate synthase -CSincreased with no differences between groups). Similar results were found by the same group (Gejl et al, 2018) when analysing glucagon and fat oxidation, higher in Low-CHO than in High-CHO, without being reflected in myocellular signalling.

Another very popular aspect nowadays is to perform fasting training, especially, depending on whether the $\mathrm{CHO}$ depletion from the previous day ("sleep-low" strategy) or the previous session ("training-low") has been reloaded. It has been described by Marquet et al (2016) how the same amount of CHO (6 g / kg) ingested but distributed differently in a High+Low training sessions week, achieve greater effects on the performance ( $2 \mathrm{~h}$ submaximal and $20-\mathrm{km}$ Time Trial), without changes in metabolic markers of the substrate used. Performing the HIIT with "high $\mathrm{CHO}$ availability" is important, but it seems that what causes the change is to complete the sessions of "low/moderate intensity" (LIT) with "low CHO availability". This protocol confirmed the changes occurred in a similar intervention that lasted 3 weeks duration (Marquet et al, 2016b), which would lead to periodise these nutritional interventions at different times of the cycling season, where there is less time margin of action.

And as Hawley and Morton (2018) pointed out, "trainlow" is one of the nutritional manipulations to promote the training response adaptation, one between few exercise-induced signalling pathways that could be sensitive to endogenous glycogen stores. One question, 
which is increasingly spreading among those involved in sports performance, is whether the benefits found in $1,4,8$ or 12 weeks interventions, will be extrapolated to more months, a season or years of duration seeking progressive adaptations.

Another special situation would be if we join the "sleep-low" with an exogenous CHO supplementation when performing sprint interval training -SIT- $(30 \mathrm{~s}$ allout +4 min active recovery) 3 days / week for 4 weeks, which Terada et al (2018) analysed. The TTE $85 \%$ increased, indicating that aerobic endurance capacity improves, but compromising peak power and total mechanical work (intensity and volume performed in SIT).

Therefore, it seems clear that we must periodize the nutritional intervention specifically according to training -continuous or intervallic-, whether low or high intensity, always looking for the "why" and "for what" (Burke et al, 2018).

The cyclist's organism will notice it. The performance will increase. The cyclist will appreciate it.

\section{Conflict of interest}

None

\section{Keywords}

Powermeters; big data; doping; road cycling; professional cycling.

${ }^{1}$ Faculty of Health Sciences, Universidad Isabel I, (Spain)

${ }^{2}$ GE-NUDAFD (Spanish Academy of Nutrition and Dietetics)

$\triangle$ Contact email: rlopezgrueso@gmail.com

\section{References}

1. Burke LM and Hawley JA (2018) Swifter, higher, stronger: What's on the menu? Science; 362(6416): 781 787.

2. Burke LM, Hawley JA, Jeukendrup A, Morton JP, Stellingwerff $\mathrm{T}$ and Maughan RJ (2018) Toward a Common Understanding of Diet-Exercise Strategies to Manipulate Fuel Availability for Training and Competition Preparation in Endurance Sport. International Journal of Sport Nutrition and Exercise Metabolism; 28(5):451-463.

3. Faria EW, Parker DL and Faria IE (2005) The science of cycling: physiology and training - part 1. Sports Medicine; 35(4): 285-312.

4. Gejl KD, Thams LB, Hansen M, Rokkedal-Lausch T, Plomgaard P, Nybo L, and Ørtenblad N (2017) No Superior Adaptations to Carbohydrate Periodization in Elite Endurance Athletes. Medicine and Science in Sports and Exercise; 49(12): 2486-2497.

5. Gejl KD, Vissing K, Hansen M, Thams L, RokkedalLausch T, Plomgaard P, and Ørtenblad N (2018) Changes in metabolism but not myocellular signaling by training with $\mathrm{CHO}-$ restriction in endurance athletes. Physiological Reports; 6(17): e13847.

6. Hawley JA and Morton JP (2014). Ramping up the signal: promoting endurance training adaptation in skeletal muscle by nutritional manipulation. Clinical and Experimental Pharmacology and Physiology; 41(8): 608-613.
7. Heikura IA, Stellingwerff T and Burke LM (2018) SelfReported Periodization of Nutrition in Elite Female and Male Runners and Race Walkers. Frontiers in Physiology; 9: 1732.

8. Heikura IA, Quod M, Strobel N, Palfreeman R, Civil R and Burke LM (2019) Alternate-Day Low Energy Availability During Spring Classics in Professional Cyclists. International Journal of Sports Physiology and Performance; 1-35. [Epub ahead of print].

9. Jeukendrup AE (2017) Periodized Nutrition for Athletes. Sports Medicine; 47(Suppl 1): 51-63.

10. Kerksick CM, Arent S, Schoenfeld BJ, Stout JR, Campbell B, Wilborn CD, and Antonio J (2017) International society of sports nutrition position stand: nutrient timing. Journal of the International Society of Sports Nutrition; 14: 33.

11. Marquet LA, Hausswirth C, Molle O, Hawley JA, Burke LM, Tiollier E and Brisswalter J (2016) Periodization of Carbohydrate Intake: Short-Term Effect on Performance. Nutrients;8(12): E755.

12. Marquet LA, Brisswalter J, Louis J, Tiollier E, Burke LM, Hawley JA and Hausswirth C (2016b) Enhanced Endurance Performance by Periodization of Carbohydrate Intake: "Sleep Low" Strategy. Medicine and Science in Sports and Exercise; 48(4): 663-72.

13. Martin MK(1), Martin DT, Collier GR and Burke LM (2002). Voluntary food intake by elite female cyclists during training and racing: influence of daily energy expenditure and body composition. International Journal of Sport Nutrition and Exercise Metabolism; 12(3): 24967.

14. Muros JJ, Sánchez-Muñoz C, Hoyos J and Zabala M (2019) Nutritional intake and body composition changes in a UCI World Tour cycling team during the Tour of Spain. European Journal of Sport Science; 19(1): 86-94.

15. Sánchez-Muñoz $\mathrm{C}$, Zabala $\mathrm{M}$ and Muros JJ (2016) Nutritional intake and anthropometric changes of professional road cyclists during a 4-day competition. Scandinavian Journal of Medicine \& Science in Sports; 26(7): 802-808.

16. Sanders D, Heijboer M (2019) Physical demands and power profile of different stage types within a cycling grand tour. European Journal of Sport Science, 19(6): 736-744.

17. Seiler KS (2010). What is best practice for training intensity and duration distribution in endurance athletes? International Journal of Sports Physiology and Performance; 5: 276-291.

18. Stellingwerff T, Morton JP and Burke LM (2019) A Framework for Periodized Nutrition for Athletics. International Journal of Sport Nutrition and Exercise Metabolism; 29(2): 141-151.

19. Stöggl TL and Sperlich B (2015) The training intensity distribution among well-trained and elite endurance athletes. Frontiers in Physiology; 6: 295.

20. Terada T, Toghi Eshghi SR, Liubaoerjijin Y, Kennedy M, Myette-Côté É, Fletcher K and Boulé NG (2019) Overnight fasting compromises exercise intensity and volume during sprint interval training but improves high-intensity aerobic endurance. The Journal of sports medicine and physical fitness; 59(3): 357-365. 